\title{
Pour en lire plus : La grande migration - Les arts plastiques pour un monde meilleur.
}

\section{Serge Manouvrier}

\section{(2) OpenEdition}

\section{Journals}

Édition électronique

URL : https://journals.openedition.org/ere/3632

DOI : $10.4000 /$ ere.3632

ISSN : 2561-2271

Éditeur

Centr'ERE

Référence électronique

Serge Manouvrier, « Pour en lire plus : La grande migration - Les arts plastiques pour un monde meilleur. », Éducation relative à l'environnement [En ligne], Volume 15 - 1 | 2019, mis en ligne le 08 octobre 2019, consulté le 17 octobre 2021. URL : http://journals.openedition.org/ere/3632 ; DOI : https://doi.org/10.4000/ere.3632

Ce document a été généré automatiquement le 17 octobre 2021

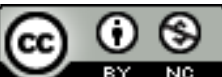

La revue Éducation relative à l'environnement est mise à disposition selon les termes de la Licence Creative Commons Attribution - Pas d'Utilisation Commerciale 4.0 International. 
Pour en lire plus : La grande migration - Les arts plastiques pour un monde meilleur.

Serge Manouvrier

RÉFÉRENCE

Deslauriers, A. (2019). La grande migration: les arts plastiques pour un monde meilleur. Montréal: JFD éditions, 270 pages. 


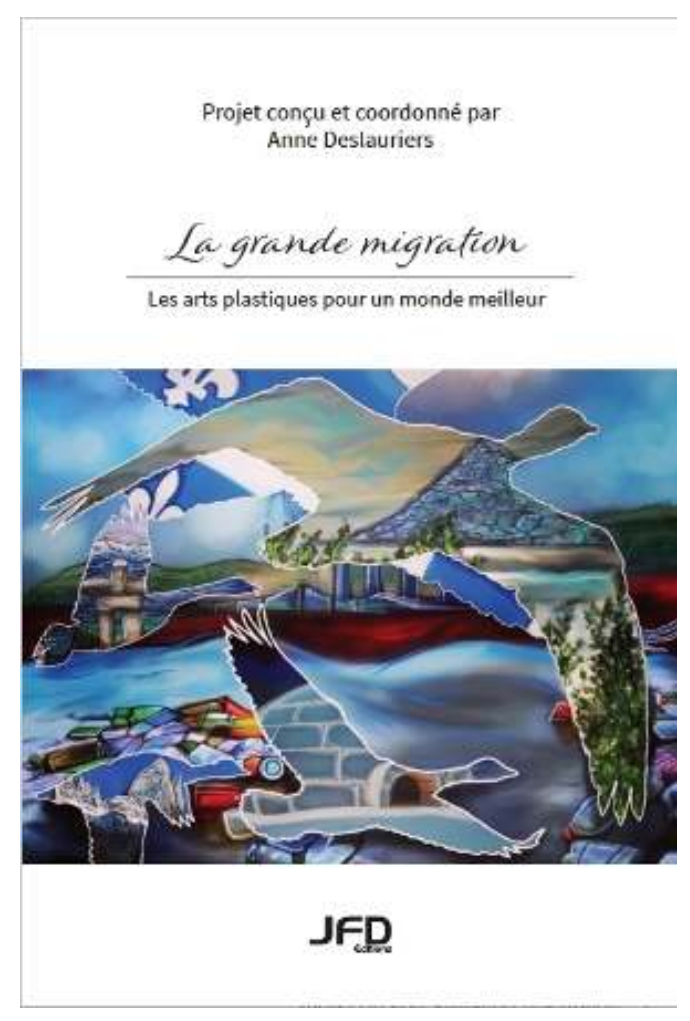

1 L'auteure de cet ouvrage, La grande migration, se situe dans la perspective de l'enseignement des arts plastiques au secondaire inspirée de la pédagogie critique pour laquelle l'éducation participe à l'émancipation de ses sujets. Partant, Anne Deslauriers prône un enseignement des arts engagé, dynamisé par des enjeux contemporains qui s'imposent avec force à notre humanité : ceux de la socioécologie. Pour ce faire, elle privilégie le recours à l'interdisciplinarité, conjuguant aux autres disciplines scolaires la transcendance et le pouvoir évocateur, voire subversif, des arts sous toutes ses formes.

2 La grande migration émane d'un projet éponyme de création interdisciplinaire autour du thème de la migration, ayant pour symbole principal la bernache. Il tente de « [servir] à lire, à dire et à s'intéresser à l'autre que l'on ne connait pas », et de "[raisonner] une dimension environnementale [chez les participants] qui ait pu renforcer leur lien d'attachement à ce monde » (p. 10). Ce projet s'est concrétisé sous l'impulsion d'Anne Deslauriers, enseignante en arts plastiques au $2^{\mathrm{e}}$ cycle du secondaire et doctorante en Études et pratiques des arts (UQAM), et par la participation de milieux formels, soit de nombreuses écoles préscolaires, primaires, secondaires et d'éducation aux adultes, ainsi que des milieux informels comme des organismes communautaires en santé mentale et en insertion sociale, et des résidences pour aînés. Ces diverses institutions proviennent de plusieurs régions du Québec, plus précisément de la grande région de Montréal et de Québec, ainsi que du Bas-Saint-Laurent et de la Côte-Nord. Le projet a impliqué directement une quinzaine d'établissements et environ 700 personnes.

3 La première partie du premier chapitre, qui correspond à la moitié de l'ouvrage, présente une série de poèmes empruntant soit à la narration, à la chanson ou au dialogue, valorisant la rime ou la prose, adoptant une facture ludique, dramatique ou contemplative. Tantôt, des préoccupations sociales sont abordées; tantôt, ce sont des préoccupations environnementales qui priment. Ici, le regard est tourné vers le passé 
ou vers un futur souhaité; là, le locuteur toise le fleuve ou scrute le ciel-horizon en contemplation de la vie. Certains poèmes témoignent de blessures et de déchirures; d'aucuns se montrent engagés, révoltés, torturés ou désabusés.

4 Le thème de la migration engendre en l'occurrence de multiples résonnances selon les lieux et contextes de production: migration humaine donnant lieu aux enjeux du déracinement et de l'acculturation; migration animale qui expose à la fois la force et la fragilité de la nature; migration temporelle qui fait ressortir les différentes phases de la vie tant au plan générationnel que personnel; migration émotive qui nous fait nous perdre dans nos propres abysses ou nous retrouver émergeant des flots...

Cette apparente hétérogénéité des poèmes, qui pourrait sembler de prime abord comme un assemblage sans fil conducteur, prend consistance pourtant à la fois par l'évocation de la bernache symbolisant le mouvement migratoire, et par un thème sous-jacent à celui de la migration qui se révèle par celui-ci, la question identitaire : l'identité ethnographique d'un Soi culturel par rapport à l'Autre; l'identité anthropologique de notre espèce dans son rapport aux autres formes de vie; l'identité psychique qui met en évidence les passages cycliques de la vie; l'identité psychologique qui nous renvoie face à nous-mêmes et qui permet d'affronter les épreuves passagères. Dans la plupart des cas, la recherche d'un avenir meilleur semble accompagner l'être et sa quête migratoire de l'ailleurs, quel qu'il soit, tissant ainsi une cohérence de propos de ce chapitre d'entrée.

6 La deuxième partie du chapitre un a trait au processus de création des bernaches, confectionnées dans des cours d'arts plastiques d'écoles du Québec et dans des centres pour aînés, et à leur périple qui converge cette fois jusqu'à la communauté autochtone de Nutashkuan, sur la Côte-Nord. On y apprend que les volatiles ont tous été marqués par leur processus migratoire discontinu. En effet, plusieurs furent victimes des affres du transport, nécessitant réparations en leurs lieux d'atterrissage. Elles subissaient alors des interventions bienveillantes de la part des créateurs qui les accueillaient pour les réexpédier presque aussitôt vers d'autres contrées scolaires ou institutionnelles.

7 Cette deuxième partie du premier chapitre se termine par la présentation du parcours migratoire de six élèves de l'école Curé-Antoine-Labelle qui ont refait le voyage des bernaches, de Laval à Nutashkuan, afin de vivre à leur tour une courte migration culturelle en territoire autochtone et les retrouvailles symboliques des bernaches qui reprendront la route vers leur terre d'origine. On y trouve des extraits de leur journal de bord transformé en véritable œuvre artistique.

Les chapitres suivants rendent essentiellement hommage aux artisans de ce projet. Le deuxième présente le portrait des différentes écoles et enseignant(e)s ayant participé au projet. Ces pages sont ponctuées de nombreuses photographies qui témoignent de l'engagement des créateurs, enfants et adultes. Le troisième chapitre souligne la contribution de deux artistes professionnels, Jean Désy et Chantal Harvey, le premier ayant œuvré en poésie à Laval, la seconde agissant comme agente culturelle pour le volet de la Côte-Nord.

9 Enfin, le quatrième chapitre rapporte le témoignage spécifique de deux enseignantes participantes : l'une par le biais de ses élèves et de leur réflexion sur le thème de la migration; l'autre par le récit de l'expérience vécue par elle-même d'un point de vue professionnel et par ses élèves d'un point de vue artistique. 
L'ouvrage La grande migration constitue en somme le témoignage d'un projet artistique ambitieux "porté par des valeurs sociales et environnementales» (p. 10) qui s'incarne dans « un geste d'ouverture, une tentative de rejoindre l'autre, de créer des liens pour un vivre-ensemble plus tangible» (p. 9). Cette aspiration éducationnelle de Anne Deslauriers concorde avec sa perspective de l'enseignement des arts inspirée par le courant de la pédagogie critique, valorisant l'engagement des élèves dans le processus de création. À la lecture de cet ouvrage, nous pouvons concevoir que cette visée de l'autrice a été atteinte. Les poèmes et les multiples photographies qui ponctuent l'ouvrage attestent d'une œuvre collective sensible hors du commun qui a assurément fait migrer ses créateurs. Quelque chose s'est passé dans ces écoles et ces institutions : une expérience artistique sociale et écologique, critique et engagée. Néanmoins et nonobstant l'envergure et la portée présumée de l'œuvre, elle incite à s'interroger quant à l'atteinte réelle des objectifs transversaux visés par de tels projets. En l'occurrence, dans quelle mesure peut-on s'assurer de la pertinence des interventions éducatives dans le développement de compétences, telle la criticité chez des sujetsélèves? Cette question nous conduit vers deux orientations porteuses de réflexions didactiques : les manifestations que nous devrions chercher à engendrer et à valoriser pour concourir à cette fin; les conditions d'enseignement et d'apprentissage qui favoriseraient l'actualisation de ces manifestations. Par conséquent, l'attention pédagogique doit migrer selon nous du produit au processus de la création et aux effets qu'elle entraine. Ces traces d'apprentissage s'avèrent toutefois éminemment plus complexes à repérer et à colliger; elles peuvent constituer un terreau fertile de recherches ultérieures, croyons-nous. La grande migration apparait à cet égard comme un ouvrage de référence pertinent qui esquisse des pistes d'investigation susceptibles d'amorcer ou de poursuivre une réflexion à la fois éducationnelle et didactique dans une perspective critique et engagée de l'enseignement des arts.

\section{AUTEUR}

\section{SERGE MANOUVRIER}

Serge Manouvrier est enseignant de français depuis plus de 15 ans au niveau secondaire. Il complète une maitrise en éducation à l'université de Sherbrooke. Ses travaux portent sur un analyseur épistémo-didactique et ses effets transformationnels sur la pratique enseignante. 